\title{
Equidos y gonfoterios del Pleistoceno tardío de San Pedro, provincia de Buenos Aires, Argentina
}

\section{Equids and Gomphotheres from the late Pleistocene of Buenos Aires province, Argentina}

\author{
J. Prado ${ }^{1}$, M.T. Alberdi²
}

\begin{abstract}
Mammal fossil remains from San Pedro (Buenos Aires Province, Argentina) are very poorly known. In this paper, best preserved specimens of Equidae and Gomphotheriidae are described. They come from "Campo Spósito" quarry near San Pedro city. The quarry was referred to Lujanian Age, Biozone of Equus (Amerhippus) neogeus. We conducted a comparative study with other South American horse and gomphothere remains, mainly from Argentina and Brazil, allow their identification as Equus (Amerhippus) neogeus, Hippidion principale and Stegomastodon cf. S. platensis. Two absolute dates were obtained by the method of OSL: $37626 \pm 4198$ y $41554 \pm 3756$ years BP. Based on this data the correlation with other localities we referred this bed to oxygen isotopic stage 3 .
\end{abstract}

Keywords: Mammals, Equidae, Gomphotheriidae, Hippidion, Equus (Amerhippus), Stegomastodon, Late Pleistocene, San Pedro, Argentina

\section{RESUMEN}

Los mamíferos fósiles de la localidad de San Pedro (provincia de Buenos Aires, Argentina) son escasamente conocidos. En este artículo se describen los ejemplares mejor conservados de Equidae y Gomphotheriidae. Estos restos provienen del yacimiento "Campo Spósito", ubicado en las proximidades de la ciudad de San Pedro. Los sedimentos portadores son referibles al Piso/Edad Lujanense, Biozona de Equus (Amerhippus) neogeus. Se realizó un estudio comparativo con caballos y gonfoterios de Argentina y Brasil para identificar los siguientes taxa: Hippidion principale, Equus (Amerhippus) neogeus y Stegomastodon cf. S. platensis. Se obtuvieron dos dataciones por Luminiscencia Ópticamente Estimulada para la secuencias estratigráfica de: $37626 \pm 4198$ y $41554 \pm 3756$ años AP, respectivamente. Estos datos sitúan el yacimiento en el Pleistoceno Superior (estadio 3 del oxígeno isotópico).

Palabras clave: Mamíferos, Equidae, Gomphotheriidae, Hippidion, Equus (Amerhippus), Stegomastodon, Pleistoceno superior, San Pedro, Argentina

\section{Introducción}

Los registros de équidos y gonfoterios en el cuaternario de la provincia de Buenos Aires son relativamente frecuentes. Muchos de estos hallazgos han sido de suma utilidad para reconocer secuencias estratigráficas portadoras de mamíferos que constituyen la escala cronológica del Cuaternario en la región (Cione et al., 2007).
Durante la década de 1990, estudios bioestratigráficos muy detallados permitieron postular que en la región Pampeana se sucedieron notables cambios en la distribución de los mamíferos vinculados a la alternancia de ambientes glaciales e interglaciares durante el Pleistoceno (Alberdi et al., 1995; Cione \& Tonni, 1995). La distribución actual de la mastofauna indica que en esta región se registra un ecotono entre la subregión Guayano-Brasileña y Patagó-

\footnotetext{
1 INCUAPA, Departamento de Arqueología. Universidad Nacional del Centro. Del Valle 5737. B7400JWI Olavarría (Argentina). Email: jprado@soc.unicen.edu.ar

2 Museo Nacional de Ciencias Naturales, CSIC. José Gutiérrez Abascal, 2. 28006-Madrid (Spain). Email: malberdi@mncn.csic.es
} 
nica (Ringuelet, 1961). Es bien conocido que durante los momentos fríos del Pleistoceno Glacial, la fauna patagónica y central invadía el área pampeana (Tonni et al., 1999). Asimismo, mamíferos habitantes de zonas abiertas se han detectado permanentemente en áreas actualmente ocupadas por selva en el norte de América del Sur (Cartelle, 1994).

Los nuevos registros y su situación estratigráfica que presentamos en este artículo permiten reinterpretar la distribución de los équidos y gonfoterios en esta región por los valiosos datos que aportan para conocer su historia biogeográfica.

El objetivo principal de este trabajo es el estudio descriptivo y sistemático de los restos de équidos y gonfoterios procedentes de la "Reserva Paleontológica Campo Spósito" en los alrededores de la ciudad de San Pedro, realizando una comparación morfométrica con los datos existentes de otras localidades en América del Sur (Alberdi et al., 1986, 1987, 1989, 2001 a, b, 2002, 2003, 2004, 2006, 2007 a, b, 2008; Ubilla \& Alberdi, 1990; Alberdi \& Prado, 1993, 2004, 2008; Alberdi \& Frassinetti, 2000; Alberdi \& Prieto, 2000; Frassinetti \& Alberdi, 2000, 2005; Paunero et al., 2008; Prado \& Alberdi, 1994; Prado et al., 1998, 2002, 2003, 2005 a, b; Rincón et al., 2006, 2009). Teniendo en cuenta la cronología de los recientes hallazgos, se analiza la distribución bioestratigráfica de estas especies.

\section{Ubicación geográfica y marco ambiental}

Los fósiles provienen de la localidad denominada "Reserva Paleontológica Campo Spósito" (33 44' 34" S 59 36' 6" O) situada en el Bajo del Tala, en las proximidades de la ciudad de San Pedro, provincia de Buenos Aires, a orillas del riachuelo Baradero que desagua al rio Paraná de las Palmas (Figura 1), que es el más meridional de los grandes brazos en que se divide el río Paraná en el tramo final. Esta zona, desde un punto de vista fisiográfico, corresponde a la Pampa Ondulada (Fidalgo, 1983) que se caracteriza, como su nombre indica, por un relieve levemente ondulado, con pendientes que van del 0,5 al 3\%. El clima es templado húmedo y según la Estación Experimental del INTA de San Pedro ( $33^{\circ} 44^{\prime} \mathrm{S}$ y $59^{\circ} 36^{\prime} \mathrm{O}$ ) la precipitación media anual en el período $1965-2002$ es de $1082,3 \mathrm{~mm}$ y la temperatura media anual de $17^{\circ} \mathrm{C}$.

El río Paraná conforma conjuntamente con los ríos Paraguay y Uruguay el sistema fluvial del río de La Plata. La cuenca del río Paraná cubre una

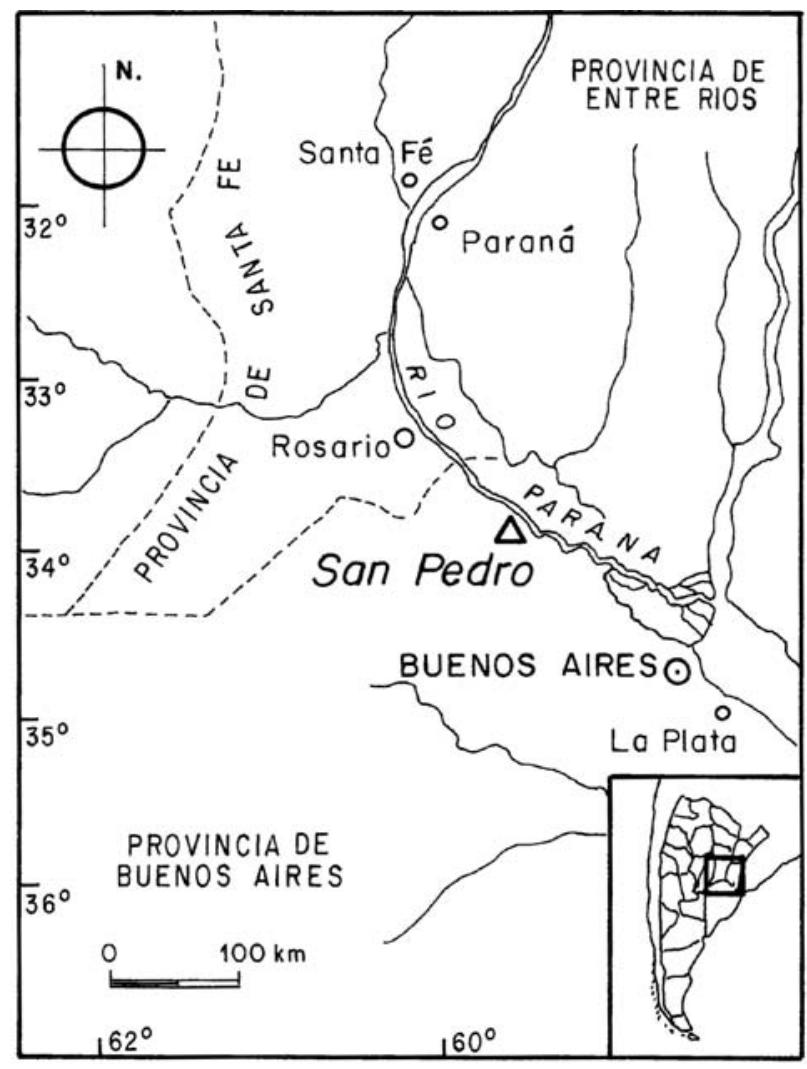

Figura 1.-Situación geográfica de la Cantera Sposito en los alrededores San Pedro (provincia de Buenos Aires, Argentina), marcada con un triángulo.

superficie de $1.510 .000 \mathrm{~km}^{2}$, en la cual se identifican tres sectores: el Paraná Superior (de la naciente a la confluencia con el río Paraguay), el Paraná Medio (de la confluencia hasta el ápice del delta) y el sector de estudio, que corresponde al Paraná Inferior (correspondiente al propio delta). Desde un punto de vista biogeográfico, en el nordeste de la provincia de Buenos Aires los mamíferos revelan una región ecotonal con elementos vinculados tanto a las selvas ribereñas (Dominio Subtropical sensu Ringuelet, 1961) como al pastizal pampeano (Dominio Pampásico).

El área de estudio se caracteriza por dos ambientes bien diferenciados, la barranca del río Paraná con su planicie de inundación y la pampa ondulada propiamente dicha hacia el interior. La planicie de inundación está caracterizada principalmente por el bosque de ribera (Distrito de las Selvas Mixtas; provincia Paranense sensu Cabrera, 1976); en tanto que la pampa ondulada se caracteriza por una estepa graminosa climáxica (distrito Pampeano Oriental de la provincia Pampeana; Cabrera, 1976) que en la 
actualidad ha sido básicamente reemplazada por cultivos, aunque se preservan relictos en los valles de inundación de los arroyos.

\section{Marco Geológico y cronológico}

Iriondo (1991) detalla que el río Paraná atraviesa en forma perpendicular o diagonal varios bloques tectónicos, lo que produce la aparición de segmentos con distintas orientaciones y pendientes locales. Dichos bloques tienen origen en las actividades tectónicas del Holoceno. Los sedimentos pleistocenos que afloran en la zona, denominados corrientemente "sedimentos pampeanos", presentan dificultades para su clasificación y correlación debido principalmente a su homogeneidad litológica. Este dato ya había sido mencionado por Castellanos (1938), en sus descripciones; pero fue originalmente d'Orbigny (1842-44) quien utilizó el término "Argille pampéene" (non vidum sensu Tonni et al., 1999) para nombrar los sedimentos marrónrojizos que cubren la mayor parte de la llanura Chaco-Pampeana. Con posterioridad, de este nombre se derivaron los términos "Formación Pampeana", "Formación Pampiano" y "sedimentos pampeanos", entre otros (Fidalgo et al., 1975). En la provincia de Buenos Aires, bajo esta denominación, se agrupa a los sedimentos asignados a las formaciones Ensenada y Buenos Aires (Riggi et al., 1986). Las dos unidades son muy similares y en algunos casos de difícil separación, por lo que actualmente a este conjunto se lo agrupa en general como sedimentos pampeanos, atribuyéndolos a los Piso/Edad Ensenadense y Bonaerense respectivamente. Son depósitos medianos a finos, limos y arcillas con intercalaciones calcáreas concrecionales o tipo mantiformes (tosca). El color dominante es el castaño, con tonalidades amarillentas a rojizas. En gran parte corresponden a sedimentos transportados por el viento desde la cordillera ya emergida para esa época y desde los llanos secos y poco vegetados que se formaron hacia el oeste de la misma. Inmensas nubes de polvo y trizas vítreas (cenizas volcánicas) de los volcanes cordilleranos alcanzaron a depositarse hasta el Atlántico, dando origen al conocido loess pampeano que fue retrabajado incesantemente por ríos, arroyos y pequeños cursos de agua, redepositándose en cauces y planicies costeras hasta la actualidad. Para la región se plantearon diferentes formas de transporte de sedimentos: eólico y fluvial (Iriondo, 1990, 1997;
Zárate \& Blasi, 1993) y diferentes áreas de aporte: Cordillera de los Andes, Sierras Pampeanas, cuenca del Paraná y escudo uruguayo (Zárate, 2003). Esta zona representa una porción de la faja periférica de loess (Iriondo \& Krohling, 1999).

En gran parte del litoral bonaerense y en la Cuenca del río de La Plata se han reconocido dos unidades que corresponden a las ingresiones marinas del Pleistoceno tardío denominadas Belgranense (Ameghino, 1889) y Formación Pascua (Fidalgo et al., 1973). La primera de ellas ha sido asignada al Ultimo Interglacial (MOIS 5e; Isla et al., 2000; Schnack et al., 2005) dentro de los esquemas más clásicos y al Holoceno la segunda (Fidalgo et al., 1973). En la zona de estudio se reconocen dos unidades que corresponderían a ingresiones marinas (Fucks \& Deschamps, 2008). La de mayor antigüedad corresponde a la Formación Pilar (Fucks \& De Francesco, 2003; Aguirre \& Fucks, 2004; Schnack et al., 2005). La más moderna, Formación Campana, corresponde a la ingresión holocena (Fucks \& De Francesco, 2003; Fucks, 2005).

En la actual red de drenaje se ha identificado una serie de unidades sedimentarias fluviales agrupadas en la Formación Luján, referida del Pleistoceno tardío al Holoceno. En algunos perfiles puede estar cubierto puntualmente por escasos metros de la Formación La Postrera, de carácter eólico y en el eje de los valles aparece cubierto por espesores más importantes de la ingresión marina holocena y depósitos fluviales (Fucks \& Deschamp, 2008).

En la localidad cercana de Baradero, Nabel et al. (1995) describieron en un perfil ocho unidades litoestratigraficas que en conjunto asignaron a las formaciones Ensenada y Buenos Aires. Las cinco inferiores eran depósitos de loess y paleosuelos alternantes y las tres superiores fueron asociadas a ambientes límnicos.

Según Fucks \& Deschamp (2008) es frecuente observar dentro de esta unidad, lentes lacustres de espesor variable y con laminaciones horizontales o estructuradas en bloques angulares pequeños. En algunos afloramientos se presentan con base cóncava y techo plano y en otros, de forma tabular.

El perfil de "Campo Spósito" presenta un depósito producto del relleno de un canal de aproximadamente $150 \mathrm{~m}$ de ancho y un espesor máximo de 1,5 $\mathrm{m}$ de donde provienen los restos fósiles de una rica fauna de mamíferos pleistocenos. Del relleno de este canal se han coleccionado además varios fragmentos de cráneos de peces dulceacuícolas asignados a armados y bagres. En este perfil (Figura 2) se 


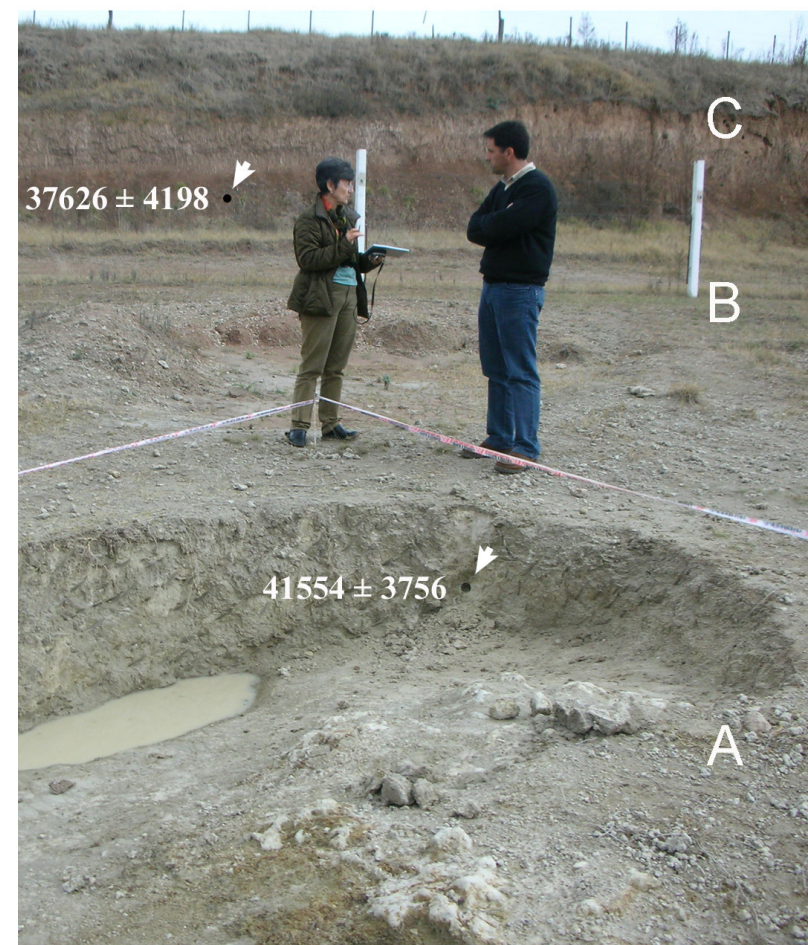

Figura 2.-Situación de las muestras para OSL en la Cantera Sposito en los alrededores de San Pedro (Buenos Aires, Argentina). A: canal con pelitas verdosas; B: Formación Buenos Aires; C: Formación La Postrera.

observan dos discordancias erosivas muy marcadas entre los depósitos asignados a la Formación Ensenada y a la Formación Buenos Aires; y entre esta última y la Formación La Postrera respectivamente. El yacimiento se encuentra en pelitas verdosas, masivas, con un conglomerado basal de calcretes rodados, rellenan un canal labrado al techo de la Formación Ensenada y son selladas por facies asimilables a la Formación Buenos Aires (Toledo, 2009). Schewenninger (in Toledo, 2009) obtuvo dos dataciones OSL de las pelitas verdes que arrojaron las siguientes edades: $30.940 \pm 2,5$ ka AP y 36.300 $\pm 2,4 \mathrm{ka} \mathrm{AP}$.

\section{Cronología}

La Edad asignada a los sedimentos pampeanos es muy variable de una región a otra. En el noreste de la provincia de Buenos Aires la mayor antigüedad corresponde al Pleistoceno temprano y está representada en los afloramientos de las «toscas del río de La Plata» (Tonni et al., 1999; Soibelzon et al.,
2008). Estudios paleomagnéticos llevados a cabo en localidades de La Plata, Buenos Aires y Baradero (Nabel, 1999; Nabel et al., 1995) sugieren unas edades magnéticas Brunhes y Matuyama. Por su parte, la antigüedad de la parte superior de estos sedimentos no esta tan clara. Hay dataciones de OSL realizadas en Baradero que indican un rango entre 114 y 14 ka (Kemp et al., 2006). Los estudios paleomagnéticos sugieren para la Formación Buenos Aires una polaridad Brunhes, asignándola al Pleistoceno medio y tardío (Nabel et al., 2000).

En el corte de "Campo Spósito", presentamos dos nuevas edades obtenidas por OSL (ver ubicación en la Figura 2). Dichas muestras fueron analizadas en el laboratorio de datación y radioquímica de la Universidad Autónoma de Madrid. Las dos muestras fueron sometidas a un test previo de decaimiento anómalo. Dicho estudio fue realizado a partir de la respuesta de OSL (sistema TL-DA-10) obtenida de las muestras en un segundo barrido, después de ser almacenadas en oscuridad durante un periodo de tiempo de 240 horas. De tal modo, que cuando las pérdidas de señal detectadas son inferiores al 3\%, dicho test se considera negativo, ó en otras palabras, el posible fenómeno de decaimiento anómalo se considera insignificante. Las pérdidas de señal detectadas en las muestras estudiadas fueron siempre inferiores al 1\%. En base a estos resultados, el método de datación seleccionado fue el de grano fino (Zimmerman, 1971), consistente en una selección de la fracción mineral con tamaño de grano comprendido entre 2-10 micras. La dosis total almacenada por cada muestra, desde que sufrió su último proceso de blanqueo solar (dosis equivalente), fue evaluada por el método de dosis aditivas. Dichas dosis crecientes fueron suministradas mediante una fuente $\mathrm{Sr}-\mathrm{Y}^{90}$ con una tasa de dosis de 0,0415 $\mathrm{Gy} / \mathrm{sg}$. Con objeto de determinar un posible comportamiento supralinear se realizó un segundo barrido, con dosis beta pequeñas (Fleming, 1970). La efectividad de las partículas alfa para producir OSL (factor K) fue determinada mediante el suministro de dosis alfa crecientes, mediante la utilización de una fuente de $\mathrm{Am}^{241}$, con una tasa de dosis de 0,0297 Gy/sg (Tabla 1). El cálculo de las dosis anuales recibidas por las muestras fue realizado mediante la combinación de dos tipos de medidas, por un lado la determinación de la radioactividad beta procedente del K-40 presente en las muestras, mediante un sistema de recuento Geiger-Müller, y por otra la medida de la actividad alfa procedente del Uranio y Torio, también presentes en las mues- 
Tabla 1.-Medidas de OSL

\begin{tabular}{lcccc}
\hline Referencia Laboratorio & Dosis Equivalente (Gy) & Supralinearidad (Gy) & Factor k & Condiciones \\
\hline MAD-5590SDA & $62,46 \pm 7,02$ & 0 & 0,02 & Tamaño grano de 2-10 micras \\
MAD-5591SDA & $83,11 \pm 7,49$ & 0 & 0,03 & \\
\hline
\end{tabular}

MAD = referencia del Laboratorio de Datación de la Universidad Autónoma de Madrid.

Tabla 2.-Resultado de las medidas de OSL

\begin{tabular}{lccc}
\hline Ref de Laboratorio & Dosis Equivalente (Gy) & Dosis Annual (mGy/año) & Números de años AP \\
\hline MAD-5590SDA & 62,46 & 1,66 & $37626 \pm 4198$ \\
MAD-5591SDA & 83,11 & 2 & $41554 \pm 3756$ \\
\hline
\end{tabular}

MAD = igual que en Tabla 1.

tras, en este caso usando un sistema de recuento de centelleo sólido $(\mathrm{ZnS})$. En este último método no se observaron perdidas de actividad como consecuencia de posibles escapes de Radón. La actividad gamma procedente de la radiación cósmica fue medida "in situ", a la toma de muestras, mediante un sistema de recuento de centelleo sólido de INa (Tl). Las conversiones de las velocidades de recuento alfa, beta y cósmica a tasa de dosis, han sido realizadas en base a los estudios de Nambi \& Aitken (1986). Los errores asociados a las edades estimadas tienen en cuenta tanto los errores sistemáticos como estadísticos correspondientes a las medidas de OSL, velocidades de dosis establecidas y procesos de calibrado de las fuentes radioactivas y equipos utilizados. El cálculo de dichos errores ha sido realizado en base a los estudios de Aitken (1985) y Arribas et al. (1990).

Los resultados obtenidos (Tabla 2), correspondientes a los sedimentos estudiados, muestran claramente el momento del último proceso de blanqueo solar sufrido por estos materiales y por tanto el momento de su formación. Las cronologías obtenidas para el nivel de pelitas verdes portador de los fósiles presentan una antigüedad de $41.554 \pm 3.756$ años AP. En tanto que el nivel inmediatamente superior a este estrato lenticular, asignado al techo de la Formación Buenos Aires, presenta una antigüedad de $37.626 \pm 4.198$ años AP.

\section{Material y método}

Los restos fósiles estudiados se encuentran depositados en las colecciones del Museo Paleontológico Municipal "Fray
Manuel de Torres" de San Pedro (provincia de Buenos Aires, Argentina) y fueron recuperados por el Sr. José Luis Aguilar y su Grupo Conservacionista de Fósiles, vecinos de la localidad. Estos se reducen a un fragmento de sínfisis mandibular rodado de équido indeterminable (GCF-sn), un fragmento de la mandíbula derecha con p2-m3 (GCF-95), dientes aislados: un P2 der (GCF-7), un P3-4 izq (GCF-130), un p2, un p3-4 y un m1-2 izquierdos (GCF-5), dos m1-2 der e izq (GCF-78), un olécranon (GCF-sn) y una primera falange del tercer digito (1FIII, GCF-sn) todos ellos de "Campo Spósito" y un metatarso del tercer dedo izq (MTIII) de Venta de Obligado (GCF-24) de Equus (Amerhippus); un M3 izq de S. Ignacio (GCF-117), un húmero der y un metacarpiano del tercer dedo der (MCIII) de "Campo Spósito" (GCF-7) de Hippidion; un fragmento de maxilar con los restos de dos M2 (MPS-8, el M2 izquierdo roto) de Cantera Biscia, un fragmento de cráneo juvenil con restos de la defensa de Cantera Biscia (MPS-73), una hemimandíbula izq con $\mathrm{m} 2$ y $\mathrm{m} 3$ de Cantera Iglesias (MPS-sn), dos fragmentos de defensa, una bastante completa de Cantera Iglesias (sin sigla) y un fragmento de Cantera fondo Avenida 3 de Febrero (MPS-11), un M1-2? izq (MPS-7) de "Campo Spósito" y un M2 izq.? de "Campo Spósito" (MPS-7) de Gomphotheriidae. Tanto los restos de Equus (Amerhippus) como los de Hippidion los hemos comparado morfológica y biométricamente con otros ejemplares procedentes de varias localidades de América del Sur depositados en distintos Museos e Instituciones. Para su descripción y análisis morfométrico, hemos seguido la nomenclatura y normas recomendadas en la "Hipparion Conference" (Eisenmann et al., 1988), así como los estudios previos realizados por Alberdi \& Prado $(1992,1993)$ y Prado \& Alberdi (1994). A fin de asignar sistemáticamente los metapodiales (un MTIII izq y una 1FIII de Equus (Amerhippus) y un MCIII izq de Hippidion), se realizó un análisis discriminante con los restos del esqueleto apendicular de las distintas especies de ambos géneros estudiados por Alberdi \& Prado (1993) y Prado \& Alberdi (1994).

En lo referente a los restos de gonfoterios se tomaron las dimensiones: longitud total (L), anchuras máximas a nivel de cada colina o lofos/lófidos (A1 a A5), y anchura del talón (T), para los dientes (ver Alberdi et al., 2002). Se han realizado análisis bivariantes y multivariantes para los dientes. Concretamente, estos datos se compararon con los restos estudiados por 
Alberdi y Prado de otras localidades de América del Sur siguiendo la metodología de Alberdi et al. (2002), tanto desde el punto de vista morfológico como biométrico. Se han realizado análisis bivariantes y análisis discriminante (AD) para los dientes, utilizando el programa SPSS versión 15.0. Asimismo, se ha aplicado el índice de robustez de Osborn (1936) para los escasos molares existentes (A máxima x 100 / L). Todas las dimensiones están expresadas en milímetros.

\section{Paleontología sistemática}

En América del Sur, la familia Equidae se encuentra representada por dos géneros: Equus (Amerhippus) Hoffstetter 1950 e Hippidion Owen 1869. En general, ambos géneros presentan especies adaptadas a los ambientes de montaña y llanura. Este grupo fue revisado por Alberdi \& Prado (1993) y Prado \& Alberdi (1994) quienes indicaron la presencia de tres especies distintas de Hippidion y cinco de Equus (Amerhippus). Los restos fósiles asignados a la familia Gomphotheriidae en América del Sur se registran desde el Pleistoceno medio hasta el Pleistoceno superior y se reconocen dos géneros distintos, Cuvieronius y Stegomastodon (Alberdi et al., 2002; Prado et al., 2002, 2005a); mientras que en el registro fósil de Argentina la presencia de esta familia está restringida al género Stegomastodon y al Pleistoceno superior.

Recientemente, Woodburne (2010) analiza el GABI (Great American Biotic Interchange) y lo divide en cuatro fases (GABI 1, 2, 3 y 4) que relaciona con las condiciones climáticas que favorecerían los intercambios faunísticos a través del istmo de Panamá. En este trabajo Woodburne anticipa la entrada de la familia Gomphotheriidae al GABI2 (en torno a $1.8 \mathrm{Ma}$ ). Si bien, en la literatura está citada la presencia de Amahuacatherium peruvium, gonfoterio del Mioceno en la Formación Madre de Dios en Perú (Campbell et al., 2000, 2001, 2010) datada en más antiguo de 9.0 Ma. Alberdi et al. (2004) consideran que los restos de Gomphotheriidae de la Formación Madre de Dios (Perú) son morfológicamente idénticos a los restos de Stegomastodon de otras localidades de América del Sur y biométricamente se encuentran claramente en la parte central de la distribución de los otros restos de m3 de gonfoterio de América del Sur. Por ello, estos autores lo consideran sinónimo de $S$. waringi presente en otras localidades de Perú. Desde el punto de vista geológico y a la vista de todos los datos indicados por Campbell et al. (2000), estos autores también consideran difícil de determinar la proveniencia exacta de los restos. En trabajos posteriores Campbell et al. $(2001,2010)$ aunque correlacionan el afloramiento con las columnas paleomagnéticas realizadas en zonas algo distantes del lugar del yacimiento situado en su entorno, se adivina complejo seguir estas correlaciones en una zona complicada geológicamente como apunta Latrubesse et al. (2007, 1010), quienes cuestionan también estas correlaciones.

\section{Orden PERISSODACTYLA Owen, 1848 \\ Familia Equidae Gray, 1821 \\ Subfamilia Equinae Gray, 1821 \\ Equus (Amerhippus) neogeus Lund, 1840}

Material. Un fragmento de mandíbula inferior izquierda con p2-m3, dos dientes superiores (un P2, un P3-4), cinco dientes inferiores (un p2, un p3-4 y tres m1-2), un olécranon, una 1FIII, y un MTIII (Figura 3).

Descripción: El fragmento de mandíbula conserva la serie de dientes yugales completas (p2-m3), le falta la sínfisis y la rama ascendente, corresponde a un animal relativamente joven. Sus dimensiones se han tomado siguiendo a Eisenmann et al. (1988): $3=94,5,4=88,6,5=194,5,10=120,11=93,12=70$. Los dientes presentan una morfología típica de Equus (Amerhippus) y su tamaño grande encaja con los restos correspondiente a Equus (A.) neogeus. La superficie oclusal de los dientes presenta el doble bucle (lazo: metacónido-metastílido) redondeado y angular, respectivamente; con el linguafléxido somero en p2 y más bien angular y algo profundo en el resto (Figura 3). El ectofléxido somero en p2, sin atravesar el istmo en p3 - p4 y m3, atravesando el istmo y casi en contacto con el linguafléxido en $\mathrm{m} 1$ y $\mathrm{m} 2$; en $\mathrm{p} 3$ - p4 y m3 el ectofléxido presenta además un pliegue caballinido más acusado en p3 y p4 que en m3. Los dientes inferiores aislados presentan los mismos rasgos morfológicos que los de la mandíbula. Dimensiones en Tabla 3.

Entre los dientes aislados superiores el P2 de tamaño grande está rodado y la morfología no se ve bien; el P3-4 también robusto con los estilos anchos y el protocono alargado con seno lingual y las fosetas escasamente plegadas. El fragmento de la ulna conserva únicamente la parte del olécranon cuya longitud es de $75,5 \mathrm{~mm}$, el estrechamiento o mínima profundidad es de $14,5 \mathrm{~mm}$ y la máxima anchura articular es $>47 \mathrm{~mm}$. La primera falange del dedo central (1FIII) y el metatarsiano del tercer dedo (MTIII) son de talla grande y con los caracteres típicos de los caballos de América del Sur (ver Prado \& Alberdi, 1994; dimensiones en la Tabla 4).

Discusión: La 1FIII se comparó con otros restos conocidos de Equus (Amerhippus) de América del Sur mediante análisis discriminante, donde se sitúa entre los restos más grandes de este género. Debido a que las primeras falanges suelen presentar una marcada variación en talla entre las anteriores y las posteriores, y que sólo disponemos de un ejemplar, este análisis no permite realizar una comparación afinada entre ellas.

Sin embargo, el MTIII se incluyó en el análisis discriminante elaborado con 74 ejemplares de Equus (Amerhippus) procedentes de distintas localidades de América del Sur (datos de Alberdi y Prado, citas en la Introducción) y queda claramente incluida entre los restos de E. (A.) neogeus. Concretamente, le aplicamos como estadístico el método de inclusión por pasos, distancias de Mahalanobis con una probabilidad F del 0,05. Los resultados indican una clasificación correcta en el 98,6\% de los casos y la validación cruzada alcanza un $95,9 \%$ de los casos correctamente identificados (Tabla 5).

E. (A.) neogeus es la especie más grande y más grácil de todas la especies de Equus (Amerhippus) de América del Sur. Se caracteriza por tener el cráneo grande y las zonas preorbital y nasal alargadas. Los huesos de las extremidades son grandes, robustos y los más gráciles de las especies de Equus (Amerhippus) conocidas en América del Sur. Tanto la morfología de los dientes como las dimensiones que tenemos de esta mandíbula coinciden con los restos conocidos de $E$. (A.) neogeus de otras localidades de América del Sur. A la vista de otros restos de mandíbulas pertenecientes a esta especie, parece que este ejemplar es algo más grande que el ejemplar de San Lorenzo (Prado \& Alberdi, 2008a), sobre todo los molares. Esto quizás responda a que este ejemplar es de una edad más joven. 


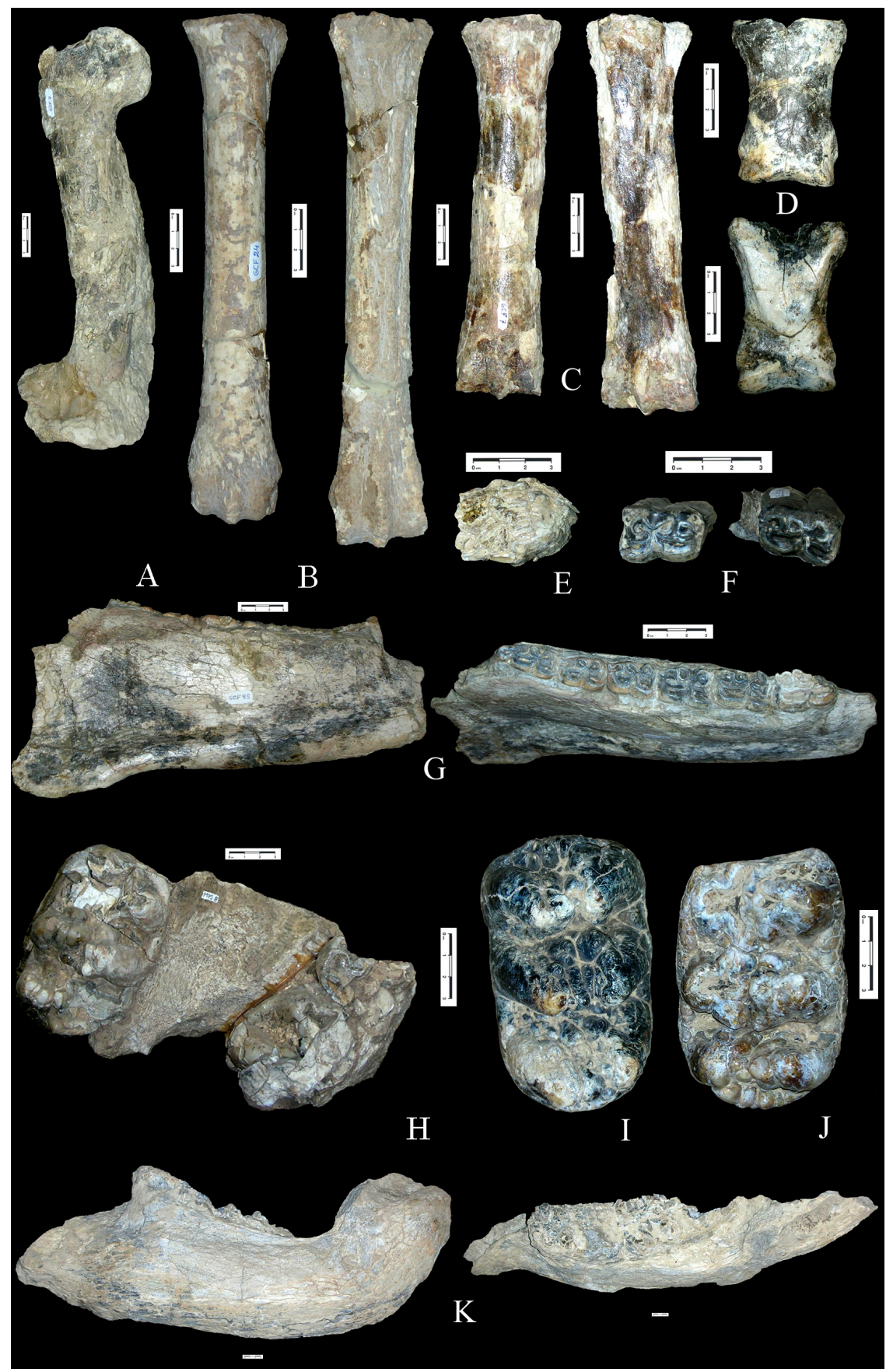

Figura 3.-Restos fósiles de de Equus (Amerhippus) neogeus Lund, 1840, Hippidion principale (Lund, 1846) y Stegomastodon cf. S. platensis procedentes de la canteras del entorno de la ciudad de San Pedro (Buenos Aires, Argentina). A: húmero derecho de $H$. principale (GCF-7) de Cantera Spósito, en vista lateral; B: MTIII izquierdo de E. (A.) neogeus (GCF-24) de Venta de Obligado, vista anterior a la izquierda y posterior a la derecha; C: MCIII de H. principale (GCF-7) de Cantera Spósito, vista anterior a la izquierda y posterior a la derecha; D: 1FIII de E. (A.) neogeus (sin número) de Cantera Spósito, vista anterior arriba y posterior debajo; E: P2 derecho de E. (A.) neogeus (GCF-7) de Cantera Spósito, en vista oclusal; F: m1-2 izquierdo y derecho de E. (A.) neogeus (GCF-78) de Cantera Spósito, en vista oclusal; G: fragmento de mandíbula derecha con p2-m3 de E. (A.) neogeus (GCF-95) de Cantera Spósito, en vista externa a la izquierda y oclusal a la derecha; $\mathrm{H}$ : fragmento de maxilar con $\mathrm{M} 2$ derecho e izquierdo de Stegomastodon cf. S. platensis (MPS-8) de Cantera Biscia, en vista ventral; I: M2 izquierdo? de Stegomastodon cf. S. platensis de Cantera Spósito, en vista oclusal; J: posible M1-2? izquierdo de Stegomastodon cf. S. platensis de Cantera Spósito, en vista oclusal; K: mandíbula izquierda con $\mathrm{m} 2$ y $\mathrm{m} 3$ de Stegomastodon cf. S. platensis de Cantera Iglesias (sin número), en vista externa a la izquierda y oclusal a la derecha. GCF = Grupo Conservacionista de Fósiles; MPS = Museo paleontológico de San Pedro. 
Tabla 3.-Dimensiones en milímetros de los dientes de Hippidion principale (Lund, 1846) y Equus (Amerhippus) neogeus Lund, 1840 procedentes del Arenero Spósito y Cantera San Ignacio, en el entorno de San Pedro (Buenos Aires, Argentina)

\begin{tabular}{|c|c|c|c|c|c|c|c|c|c|c|c|}
\hline Sigla & Género & Ciente & Localidad & Ls & As & $\mathrm{Lb}$ & $\mathrm{Ab}$ & $\mathrm{H}$ & Lpr & Postflex. & Lazo \\
\hline GCF-5 & Equus & p2 izq & Arenero Sposito & & 18 & & & 35 & & 15,2 & \\
\hline GCF-5 & Equus & p3-4 izq & Arenero Sposito & 30 & 20 & & & 40 & & 15 & 17,6 \\
\hline GCF-5 & Equus & m1-2 izq & Arenero Sposito & & & & & 61 & & 13,7 & \\
\hline GCF-78 & Equus & m1-2 der & Arenero Sposito & 27,2 & 17,2 & 22 & 15 & 60 & & 11,3 & 14 \\
\hline GCF-78 & Equus & m1-2 izq & Arenero Sposito & 26,5 & 16 & 24,8 & 15,1 & 61 & & 11,5 & 13,5 \\
\hline GCF-7 & Equus & $\mathrm{P} 2$ der & Arenero Sposito & 40,5 & 30,5 & & & 48 & 10,1 & & \\
\hline GCF-130 & Equus & P3-4 izq & Arenero Sposito & 30 & 31,8 & 25,2 & 30,7 & 75 & 15,6 & & \\
\hline GCF-95 & Equus & p2 der & Arenero Sposito & 34 & 22,3 & & & en mand & & 18 & 18,7 \\
\hline GCF-95 & Equus & p3 der & Arenero Sposito & 31,2 & 22,2 & & & en mand & & 15 & 16,5 \\
\hline GCF-95 & Equus & p4 der & Arenero Sposito & 29 & 22 & & & en mand & & 14,2 & 15,6 \\
\hline GCF-95 & Equus & m1 der & Arenero Sposito & 26,8 & 18,8 & & & en mand & & 9,8 & 15 \\
\hline GCF-95 & Equus & $\mathrm{m} 2 \mathrm{der}$ & Arenero Sposito & 27,9 & 17,8 & & & en mand & & 9,6 & 13 \\
\hline GCF-95 & Equus & $\mathrm{m} 3 \mathrm{der}$ & Arenero Sposito & 33 & 17 & & & en mand & & 12,5 & 12,6 \\
\hline GCF-117 & Hippidion & M3 izq & Cantera S Ignacio & 28,5 & 28 & 28 & 28,6 & 38 & 10,1 & & \\
\hline
\end{tabular}

Ls, longitud mesio-distal en superficie oclusal; As, anchura linguo-bucal en superficie ocusal; $H$, altura del diente; Lpr, Longitud mesio-distal del protocono en superficie oclusal; postflex., longitud mesio-distal del postfléxido; lazo, longitud del lazo metacónidometastílido en superficie oclusal. P/p, premolares superiores/inferiores; $\mathrm{M} / \mathrm{m}$, molares superiores/inferiores. GCF = Grupo Conservacionista de Fósiles.

Tabla 4.-Dimensiones en milímetros de los huesos de Hippidion principale (Lund, 1846) y Equus (Amerhippus) neogeus Lund, 1840 procedentes del Arenero Spósito y Venta Obligado, en el entorno de San Pedro (Buenos Aires, Argentina)

\begin{tabular}{|c|c|c|c|c|c|c|c|c|c|c|c|}
\hline Sigla & Género & Hueso & Localidad & $\begin{array}{c}\text { Medida } \\
1\end{array}$ & $\begin{array}{c}\text { Medida } \\
2\end{array}$ & $\begin{array}{c}\text { Medida } \\
3\end{array}$ & $\begin{array}{c}\text { Medida } \\
4\end{array}$ & $\begin{array}{c}\text { Medida } \\
5\end{array}$ & $\begin{array}{c}\text { Medida } \\
6\end{array}$ & $\begin{array}{c}\text { Medida } \\
7\end{array}$ & $\begin{array}{c}\text { Medida } \\
8\end{array}$ \\
\hline GCF-sn & Equus & 1FIII & Arenero Sposito & 81 & 66 & 38 & 53 & 34 & 43,5 & 45 & 27 \\
\hline GCF-7 & Hippidion & McIII izquierdo & Arenero Sposito & 208 & 201 & 40,41 & 29 & 58 & 41,5 & 50 & 12 \\
\hline GCF-24 & Equus & MtIII izquierdo & Venta de Obligado & $>258$ & $>250$ & 32,4 & 33,2 & 53 & 45 & 47,7 & 11,7 \\
\hline GCF-7 & Hippidion & Húmero derecho & Arenero Sposito & & 294 & 43,5 & 63 & 102 & $>115$ & 92 & 92,5 \\
\hline GCF-sn & équido & olécranon & Arenero Sposito & & $>75,5$ & $>14,5$ & & $>47$ & & & \\
\hline Sigla & Género & Hueso & Localidad & $\begin{array}{c}\text { Medida } \\
9\end{array}$ & $\begin{array}{c}\text { Medida } \\
10\end{array}$ & $\begin{array}{c}\text { Medida } \\
11\end{array}$ & $\begin{array}{c}\text { Medida } \\
12\end{array}$ & $\begin{array}{c}\text { Medida } \\
13\end{array}$ & $\begin{array}{c}\text { Medida } \\
14\end{array}$ & $\begin{array}{c}\text { Medida } \\
16\end{array}$ & \\
\hline GCF-sn & Equus & 1FIII & Arenero Sposito & 45 & & & & & & & \\
\hline GCF-7 & Hippidion & McIII izquierdo & Arenero Sposito & 9,2 & 50,8 & 50 & 37,7 & 31 & 34,4 & 10 & \\
\hline GCF-24 & Equus & MtIII izquierdo & Venta de Obligado & 9,7 & 49,3 & 47,6 & 31,2 & 28,5 & 32 & & \\
\hline GCF-sn & équido & olécranon & Arenero Sposito & & & & & & & & \\
\hline
\end{tabular}

Dimensiones siguiendo las recomendaciones de la "Hipparion Conference" Eisenmann et al., 1988. Dimensiones en milímetros. Sn $=$ sin número; GCF = igual que en Tabla 3 .

Hippidion Owen, 1869

Hippidion principale (Lund, 1846)

Material: solamente un M3, un húmero y un MCIII.

Descripción: El único molar disponible es un M3 bastante gastado con morfología oclusal primitiva, tipo Pliohippus, característica de este género. Este diente es robusto con el protocono oval-alargado, el hipocono incluido en el lofo y el desgaste inclinado distalmente. Sus dimensiones son: 28,5 x 28 en superficie oclusal y 28 x 28,6 a $1 \mathrm{~cm}$ de la base, 38 de altura y
10,1 de longitud del protocono en superficie oclusal. En los análisis bivariantes se sitúa entre los dientes de mayor tamaño correspondientes a Hippidion principale. El húmero tiene la superficie bastante alterada, sus dimensiones en Tabla 4. Es similar en talla a los húmeros estudiados en Miramar y Tarija de tamaño grande (Figura 3).

El MCIII es un ejemplar completo y relativamente corto y ancho, con un índice de gracilidad característico del género Hippidion ( $\mathrm{IG}=16,13)$. Las dimensiones están en la Tabla 4. La morfología del hueso, incluidas las epífisis, está algo alterada (Figura 3). En los análisis bivariantes este metacarpiano se 
Tabla 5.-Resultados de los análisis discriminantes del MTIII de Venta Obligado comparado con 74 metatarsianos de distintas especies de Equus (Amerhippus) de América del Sur; y del MCIII de Cantera Spósito comparado con 35 metacarpiano de distintas especies de Hippidion de América del Sur. Más explicación en el texto

\begin{tabular}{|c|c|c|c|c|c|c|}
\hline & & E. (A.) neogeus & E. (A.) insulatus & E. (A.) andium & E. (A.) santaeelenae & Totales \\
\hline \multirow[t]{6}{*}{ Recuento original } & E. (A.) neogeus & 12 & 0 & 0 & 0 & 12 \\
\hline & E. (A.) insulatus & 0 & 14 & 0 & 1 & 15 \\
\hline & E. (A.) andium & 0 & 0 & 39 & 0 & 39 \\
\hline & E. (A.) santaeelenae & 0 & 0 & 0 & 7 & 7 \\
\hline & MTIII de San Pedro & 1 & 0 & 0 & 0 & 1 \\
\hline & & H. principale & H. devillei & H. saldiasi & & Totales \\
\hline \multirow[t]{4}{*}{ Recuento original } & H. principale & 11 & 0 & 0 & & 11 \\
\hline & H. devillei & 2 & 11 & 3 & & 16 \\
\hline & H. saldiasi & 0 & 0 & 7 & & 7 \\
\hline & MCIII de San Pedro & 1 & 0 & 0 & & 1 \\
\hline
\end{tabular}

sitúa en la zona de contacto entre los correspondientes a $H$. principale e $H$. devillei.

Discusión: Los dientes se incluyeron en el análisis discriminante elaborado con los dientes estudiados por nosotros de otras localidades de América del Sur. En este análisis los dientes se sitúan entre los restos de $H$. principale con un porcentaje de clasificación correcta del $66,7 \%$ y ésta pasa al $50,0 \%$ con validación cruzada. Debe tenerse en cuenta que los dientes son, en general, muy variables debido a que al ser dientes hipsodontos éstos varían según los distintos grados de desgaste en los que se pueden encontrar. En el caso del MCIII, se incluyó en el análisis discriminante elaborado con 35 ejemplares de MCIII de Hippidion procedentes de distintas localidades de América del Sur (datos de Alberdi y Prado, citas en la Introducción), quedando claramente incluido entre los restos de $H$. principale. Concretamente, si le aplicamos como estadístico el método de inclusión por pasos, distancias de Mahalanobis, los resultados indican una clasificación correcta en el $85,3 \%$ de los casos y la validación cruzada alcanza un $82,4 \%$ de los casos correctamente identificados (Tabla 5).

A pesar de los escasos restos de Hippidion presentes en esta localidad, la morfología y el tamaño de los ejemplares permite asignarlos a $H$. principale gracias a la base de datos, material estudiado de otras localidades de América del Sur, de la que disponemos y que nos ha permitido contrastar todas sus características morfológicas y biométricas (ver citas en la Introducción). Esta especie es la forma más grande de todos los hipidiformes conocidos.

Orden PROBOSCIDEA Owen, 1848

Familia Gomphotheriidae Cabrera, 1929

Genero Stegomastodon Pohlig, 1912

Stegomastodon cf. S. platensis Ameghino (1888)

Material: Estos se reducen a un fragmento de cráneo con restos de defensa (MPS-73), dos fragmentos de defensa (MPS11, MPS-sn), un fragmento de maxilar con los restos de dos M2 (MPS-8), dos dientes aislados superiores: un M1-2? y un
M2 (MPS-7), y una hemimandíbula izquierda con m2 y m3 (MPS-sn).

Descripción: Los restos de gonfoterios procedente de "Campo Spósito", Cantera Iglesias y Cantera Biscia son muy escasos y la mayoría están algo alterados. Una defensa tiene 2,26 m de longitud y está ligeramente curvada, su sección máxima en la base es de 182 x $153 \mathrm{~mm}$, la sección a la mitad de su longitud es de 160 x 147, y en la zona del ápice es de 80 x 59, esta defensa es característica de Stegomastodon. El otro fragmento de defensa mide $100,10 \mathrm{~cm}$ de longitud y su diámetro en la parte más ancha es de 14,7 x $11 \mathrm{~cm}$. El fragmento de maxilar conserva los dos M2 bastante grandes, el derecho está mejor conservado que el izquierdo, con línea media clara, cemento abundante, no muy desgastado, primera colina algo rota en el derecho, el izquierdo conserva sólo la mitad del diente, dimensiones en Tabla 6. Además, disponemos de dos dientes aislados, un posible M1-2? y un M2 cuyas dimensiones permitirán compararlos con la base de datos de Alberdi y Prado (ver referencias en la Introducción), si bien los dientes de los gonfoterios son muy variables. Los M2 presentan tres colinas (o lofos), con línea media clara, talón reducido, apuntando un posible desgaste de doble trébol (dimensiones en Tabla 6). La hemimandíbula izquierda conserva $\mathrm{m} 2$ y $\mathrm{m} 3$, la sínfisis algo recurvada hacia abajo y el inicio de la rama ascendente. Las dimensiones de la mandíbula, siguiendo los caracteres morfológicos y parámetros seleccionados en Alberdi et al. (2002), son: 6- borde mandibular está muy alterado; 7- la longitud de la serie dentaria $=352,5 \mathrm{~mm} ; 8$ - presenta un foramen mentoniano anterior al m2; 9- la sínfisis mandibular recurvada y también alterada; a- longitud máxima en el plano sagital aproximada de $670 \mathrm{~mm}$; b- altura de la mandíbula a nivel anterior del $\mathrm{m} 2=$ $210 \mathrm{~mm}$; c- altura de la mandíbula a nivel posterior del $\mathrm{m} 3=$ $163 \mathrm{~mm}$. El m2 está muy desgastado y la superficie alterada (en el sentido tafonómico del término) y tiene una longitud máxima en torno a $156 \mathrm{~mm}$, el $\mathrm{m} 3$ está igualmente alterado y roto y tiene una longitud máxima en torno a $227 \mathrm{~mm}$.

Discusión: Los M2 han sido incluidos en un diagrama bivariante elaborado con los datos de $44 \mathrm{M} 2$ superiores de distintas localidades de América del Sur que dejan clara la gran variabi- 
Tabla 6.-Dimensiones en milímetros de los M2 de Stegomastodon cf. S. platensis (Ameghino, 1888) procedentes del Arenero Spósito, Cantera Iglesias y Cantera Biscia en el entorno de San Pedro (Buenos Aires, Argentina)

\begin{tabular}{|c|c|c|c|c|c|c|c|c|c|}
\hline Sigla & Diente/hueso & Localidad & $\mathrm{L}$ & A1 & A2 & A3 & A4 & A5 & $A>$ \\
\hline MPS-7 & M2 izquierdo & Arenero Sposito & 114 & 61 & 70 & 70 & & & \multirow[b]{3}{*}{ reducido } \\
\hline MPS-7 & M1 izquierdp & Arenero Sposito & 93 & 64,3 & 67 & 64 & & & \\
\hline MPS-8 & M2 derecho & Cantera Biscia & 117 & 71 & 75 & 71 & & & \\
\hline MPS-8 & $\begin{array}{l}\text { fragmento } \\
\text { maxilar }\end{array}$ & Cantera Biscia & $\begin{array}{l}\text { figuras perdidas } \\
\text { por desgaste }\end{array}$ & & & & & & \\
\hline MPS-73 & $\begin{array}{l}\text { frag. maxilar } \\
\text { juvenil }\end{array}$ & Cantera Biscia & restos de defensa & & & & & & \\
\hline sin número & $\mathrm{m} 2$ left & Cantera Iglesias & 156 & & & & & & \\
\hline sin número & m3 left & Cantera Iglesias & 227 & & & & & & \\
\hline MPS-11 & tusk & Cantera fondo & & & & & & & \\
\hline sin número & & Avda 3 de febrero & 1000,1 & $\begin{array}{l}>\mathrm{db}=147 \\
>\mathrm{dms}=18 ?\end{array}$ & $<\mathrm{db}=110$ & & & & \\
\hline sin número & tusk & Cantera Iglesias & 2260 & $>\mathrm{dms}=182$ & $\mathrm{dms}=153$ & $>\mathrm{da}=80$ & $\mathrm{da}=59$ & & \\
\hline
\end{tabular}

Parámetros seleccionados siguiendo Alberdi et al. (2002): L: longitud máxima; A1, A2, A3, A4 y A5: anchuras máximas de los respectivos lofos/lófidos; T: talón; $A>$ : máxima anchura; >db: diámetro mayor en la base; $<\mathrm{db}=$ diámetro.

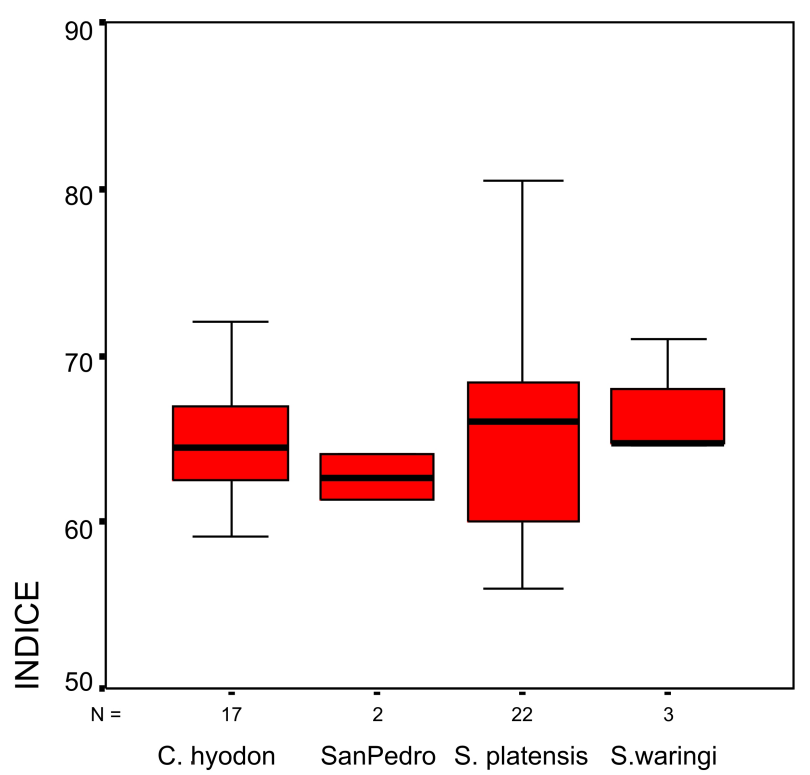

ESPECIE

Figura 4.-Diagrama de caja de los índices de anchura de los M2 de Stegomastodon procedentes uno de Cantera Spósito y otro de Biscia del entorno de la ciudad de San Pedro (provincia de Buenos Aires, Argentina), siguiendo Osborn (1936) comparado con los correspondientes índices de anchura de los restos de M2 de otras localidades de América del Sur.

lidad de estos elementos dentarios. Los restos del área de San Pedro se encuentran entre los dientes más pequeños tanto de Stegomastodon como de Cuvieronius. Los diagramas de caja elaborados a partir de los índices de anchura de los molares extraídos siguiendo a Osborn (1936) indican que estos dientes se sitúan en el intervalo de los molares de Stegomastodon más que en los de Cuvieronius, pero sin gran precisión (Figura 4). Los datos morfológicos de los molares (tipo bunodonto con conulos centrales y línea media marcada) y los análisis estadísticos, indicados más arriba, sugieren que los restos dentarios estudiados no son significativos a la hora de identificar estos restos. En los diagramas bivariantes éstos se sitúan entre los restos más pequeños de Cuvieronius y Stegomastodon, debido a la gran variabilidad de los molares de los gonfoterios y a la escasez de los mismos. Los diagramas de caja elaborados a partir de los índices de anchura de Osborn están más en la distribución de Stegomastodon platensis que en la de Cuvieronius hyodon (Figura 4). La defensa prácticamente completa, procedente de Cantera Iglesias, presenta una morfología claramente identificable con el género Stegomastodon, ligeramente curva sin banda de esmalte y de tamaño grande.

\section{Discusión y conclusiones}

El nombre de Hippidion, creado por Owen en 1869, junto con el de Equus (Amerhippus), fue citado en numerosas localidades de Argentina y otros países de América del Sur (ver citas en la Introducción). El primer representante de Hippidion fue descrito a partir de un único molar de una brecha antigua de la Cueva de Lagoa Santa (Brasil) y figurado por Lund en 1846. Alberdi \& Prado (1993) y Prado \& Alberdi (1996) consideran que este género es endémico en América del Sur donde se expande desde el Plioceno superior al Pleistoceno final (Alberdi \& Prado, 1993) y geográficamente se distribuye en gran parte de América del Sur, principalmente en Argentina, Bolivia, Brasil, Chile, Colom- 
bia, Perú y Uruguay (ver citas en la Introducción). El primer representante de Equus fue recuperado por Darwin en Bahía Blanca durante la expedición del Beagle y descrito por Owen (1840).

La revisión taxonómica de los géneros Hippidion y Equus (Amerhippus) fue llevada a cabo por Alberdi \& Prado (1993) y Prado \& Alberdi (1994), respectivamente, mediante análisis multivariantes sobre variables cuantitativas de los restos más significativos del esqueleto apendicular. Estos análisis indicaron la presencia de tres especies distintas en el caso del género Hippidion y cinco en el caso de Equus (Amerhippus).

Luego de las comparaciones y los análisis realizados podemos concluir que los restos de équidos procedentes del área de San Pedro, son determinados como Equus (Amerhippus) neogeus e Hippidion principale.

Por su parte, los restos de gonfoterios asociados con los équidos mencionados son escasos. La morfología y tamaño de la mandíbula (MPS-sn), a pesar de estar muy alterada, parece indicar su pertenencia a un animal relativamente pequeño en talla, incluso los molares también son pequeños. Los caracteres de los dientes aislados no permiten una determinación precisa por estar bastante alterados. Sin embargo, las dos defensas (MPS-sn y MPS-11), una prácticamente entera y un fragmento de otra sin torsión helicoidal y carente de esmalte, indica que nos encontramos ante un ejemplar del género Stegomastodon.

Los análisis morfométricos de los restos dentarios y los análisis bivariantes de los M2 sólo indican que nos encontramos ante un individuo relativamente pequeño que podría corresponder a la especie S. platensis por apuntar en la superficie oclusal de uno de los molares una complicación mayor del dibujo del esmalte, dando figuras treboladas dobles a ambos lados de la línea media. No obstante este carácter tampoco es taxativo (Alberdi et al., 2002, 2004, 2007b; Prado et al., 2002; Prado \& Alberdi, 2008b). Hasta el presente todos los ejemplares coleccionados en la provincia de Buenos Aires han sido incluidos en el género Stegomastodon y en caso de determinaciones específicas han sido asignados a Stegomastodon platensis. Recientemente, se ha mencionado el hallazgo de una rama mandibular incompleta en el lecho del Río de La Plata referida a Stegomastodon waringi (Cione et al., 2005). Esta cita, equidistante de las costas de Argentina y Uruguay, es el registro más austral de esta especie y concuerda con los hallaz- gos recientes en el territorio uruguayo donde sólo hemos encontrado restos asignados a Stegomastodon waringi (Gutiérrez et al., 2005; Alberdi et al., 2007b).

Por otra parte, consideramos que esta asociación y los yacimientos del área de San Pedro revisten un gran interés desde un punto de vista bioestratigrafico y paleoambiental. Tanto las dos especies de caballo citadas como el Stegomastodon son especies frecuentes en el Piso/Edad Lujanense. Cione \& Tonni (1999) sustentan, desde un punto de vista bioestratigrafico, el Lujanense (non Ameghino, 1889; non Pascual et al., 1965) por la biozona de Equus (Amerhippus) neogeus. Los datos y la cronología aportada en este artículo apoyan la presunción de que esta biozona se extiende hasta el periodo isotópico OIS 3. Asimismo, la presencia conjunta de ambas especies de caballos ha sido mencionada en Uruguay, en las facies conglomeráticas basales de la Formación Sopas, que fueron correlacionadas por Toledo (2005) con los depósitos de "Campo Spósito". Para la Formación Sopas se ha obtenido por OSL una edad de $43 \pm 3,6 \mathrm{ka}$ a $58,3 \pm 7,4 \mathrm{ka}$ (Ubilla, 2004) y por consiguiente también referida al estadio isotópico OIS 3. Si bien esta asociación no es muy frecuente, también se la ha mencionado en un entorno cercano, en la localidad San Lorenzo (provincia de Santa Fé, Prado \& Alberdi, 2008a). En este caso no fue posible una cronología radiométrica y se refirieron los niveles portadores al último glacial. Otra localidad en la provincia de Santa Fé que presenta la misma asociación de caballos y gonfoterios está en las márgenes del río Carcarañá, de donde ya Darwin recuperó restos de gonfoterios y en las colecciones del Museo "Bernardino Rivadavia" de Buenos Aires se encuentra una notable colección de caballos y gonfoterios procedentes de la Formación Carcarañá. Estos niveles han sido datados recientemente mediante OSL en una edad entre 45,6 $\pm 1,9 \mathrm{ka}$ y 52,3 $\pm 1,2 \mathrm{ka}$ (Krohling, 1999). Esta asociación de Equus (Amerhippus) neogeus e Hippidion principale también se ha registrado en el Bonaerense de la provincia de Buenos Aires, en la localidad de Mercedes (Alberdi \& Prado, 2004) y en Arroyo Tapalqué en torno a $38^{\circ}$ S (Prado et al., 2011). Los datos de esta última localidad son interesantes ya que tenemos información de la dieta inferida a partir de isotopos estables de Carbono. Los valores de $\delta^{13} \mathrm{C}$ para $H$. principale $(-11,6$ a $-9,3 \%$ ) y $E$. (A.) neogeous $(-8,5$ a $-7,9 \%$ o) no se solapan, pero ambos están bastante cerca. El rango de valores de $H$. principale corresponde a 
una dieta de plantas $\mathrm{C}_{3}$, mientras que $E$. (A.) insulatus correspode a una dieta de plantas $\mathrm{C}_{3} \mathrm{y} \mathrm{C}_{4}$, es decir mixta. Por su parte, si nos fijamos en la morfología, estos taxones son muy diferentes. La morfología del cráneo y las extremidades nos sugieren que estas formas presentan distintas adaptaciones. En general, los dientes de Equus son más hipsodontos que los de Hippidion y el patrón de esmalte es más complicado en Equus. Otra diferencia radica en el tamaño corporal, su masa corporal inferida a partir de una regresión alométrica es de 460 y 378 $\mathrm{kg}$, respectivamente (Alberdi et al., 1995). El cráneo de Equus (Amerhippus) neogeus es grande y muestra una región preorbital y nasal amplia. Los huesos de las extremidades son grandes y robustos, pero más delgado que en las otras especies de América del Sur (Alberdi \& Prado, 1992, 1993; Prado \& Alberdi, 1994). En tanto que Hippidion principale presenta una retracción de la hendidura nasal que permite inferir una probóscide. El esqueleto es grande y masivo, con extremidades fuertes, sobre todo los metapodos y las falanges. Es la especie más grande y robusta de Hippidion. Estas características han permitido inferir el tipo de alimentación y el hábitat. La morfología de $H$. principale sugiere que puede ser un ramoneador, pero que puede adaptarse a vivir en praderas abiertas. Por su parte, E. (A.) neogeus es más hipsodonto, y tienen el característico hocico estrecho de las formas pastadoras de pastos $\mathrm{C}_{4}$ (Alberdi \& Prado 2004), pero en estas latitudes tolerarían una dieta mixta de pastos $\mathrm{C}_{3} \mathrm{y} \mathrm{C}_{4}$. Esta observación coincide con los datos obtenidos por MacFadden et al. (1999) sobre el gradiente latitudinal observado en la distribución de Equus en el Pleistoceno de ambas Américas. Los valores de $\delta^{13} \mathrm{C}$ parecen ser simétricos a ambos lados del ecuador. En América del Norte, la transición entre una señal isotópica $\mathrm{C}_{4}$ y $\mathrm{C}_{3}$ plena se observa alrededor de $45^{\circ}$ latitud $\mathrm{N}$ y en torno a $35^{\circ}$ latitud $\mathrm{S}$ en América del Sur (provincia de Buenos Aires).

En síntesis, en estas latitudes, esta asociación de Equus (Amerhippus) neogeus, Hippidion principale y Stegomastodon es característica del estadio isotópico OIS 3 (59-28 Ka). Durante este estadio el clima global es en general frío pero presenta un patrón climático más complejo, con algún intervalo más cálido que permitió la coexistencia en estas latitudes de estas especies. Iriondo (1994, 1999) propuso un rápido cambio del clima de las condiciones frías y secas del OIS 4 hacia condiciones más húmedas y cálidas en el OIS3. Muy posible- mente la existencia de ambientes mixtos en estas localidades del noroeste de las provincias de Buenos Aires y Santa Fé, en latitudes entre 32 a $33^{\circ}$ esté relacionada con condiciones climáticas locales más benignas durante el último glacial, ya que en las localidades por debajo de los $34^{\circ}$ de latitud Sur solamente se registra en el Pleistoceno tardío Equus (Amerhippus) neogeus.

\section{AGRADECIMIENTOS}

Queremos agradecer especialmente a José Luis Aguilar responsable del Museo Paleontológico Municipal "Fray Manuel de Torres" de San Pedro (provincia de Buenos Aires, Argentina) por haber puesto a nuestra disposición este material y todas las facilidades para su estudio. Al equipo de colaboradores y entusiastas de la paleontología en San Pedro, que llevaron adelante las tareas de campo y restauración de los fósiles. También queremos agradecer al Dr. Marcelo Toledo por sus valiosos comentarios sobre la estratigrafía de la zona. Igualmente a Martín Ubilla y un revisor anónimo cuyos comentarios y recomendaciones han mejorado considerablemente el trabajo. Este trabajo se ha realizado en el marco de los Proyectos CGL200760790/BTE de la DGCYT y A/023681/09 de la AECID, España, y las Subvenciones de la Universidad Nacional del Centro de la Provincia de Buenos Aires y el Proyecto ANPCYT PICT 07-01563, Argentina.

\section{Referencias}

Aguirre, M. \& Fucks, E. (2004). Moluscos y Paleoambientes del Cuaternario Marino en el sur de Entre Ríos y Litoral Bonaerense. In: Temas de la Biodiversidad del Litoral Fluvial Argentino (Aceñolaza, F., ed.). INSUGEO, Miscelanea, 12: 55-70.

Aitken, M.J. (1985). TL Dating. Academy Press, London Alberdi, M.T. \& Prado, J.L. (1992). El registro de Hippidion Owen, 1869 y Equus (Amerhippus) Hoffstetter, 1950 (Mammalia, Perissodactyla) en América del Sur. Ameghiniana, 29: 265-284.

Alberdi, M.T. \& Prado, J.L. (1993). Review of the genus Hippidion Owen, 1869 (Mammalia; Perissodactyla) from the Pleistocene of South America. Zoological Journal of the Linnean Society, 108: 1-22. doi:10.1111/j.1096-3642.1993.tb02559.x

Alberdi, M.T. \& Prado, J.L. (2004). Caballos fósiles de América del Sur. Una historia de tres millones de años. INCUAPA Serie Monográfica, 3: 1-269.

Alberdi, M.T. \& Prado, J.L. (2008). Presencia de Stegomastodon (Gomphotheriidae, Proboscidea) en el Pleistoceno superior de la zona costera de Santa Clara del Mar (Argentina). Estudios geológicos, 64(2): 175-185.

Alberdi, M.T. \& Frassinetti, D. (2000). Presencia de Hippidon y Equus (Amerhippus) (Mammalia, Perissodactyla) y su distribución en el Pleistoceno Superior de Chile. Estudios Geológicos, 56: 279-290. 
Alberdi, M.T. \& Prieto, A. (2000). Hippidion (Mammalia, Perissodactyla) de las Cuevas de las provincias de Magallanes y Tierra de Fuego. Anales del Instituto de la Patagonia, Serie Ciencias Humanas (Chile), 28: 147-171.

Alberdi, M.T.; Fernández, J.; Menegaz, A.N. \& Prado, J.L. (1986). Hippidion Owen 1869 (Mammalia, Perissodactyla) en sedimentos del Pleistoceno tardío de la localidad Barro Negro (Jujuy, Argentina). Estudios geológicos, 42: 487-493.

Alberdi, M.T.; Menegaz, A.N. \& Prado, J.L. (1987). Formas terminales de Hippidion (Mammalia, Perissodactyla) de los yacimientos del Pleistoceno tardío Holoceno de la Patagonia (Argentina y Chile). Estudios Geológicos, 43: 107-115.

Alberdi, M.T.; Menegaz, A.N.; Prado, J.L. \& Tonni, E.P. (1989). La fauna local de Quequén Salado-Indio Rico (Pleistoceno tardío) de la provincia de Buenos Aires, Argentina. Aspectos paleoambientales y biostratigráficos. Ameghiniana, 25: 225-236.

Alberdi, M.T.; Leone, G. \& Tonni, E.P. (1995). Evolución Biológica y Climática de la Región Pampeana durante los últimos cinco millones de años. Un ensayo de correlación con el Mediterráneo occidental. Monografías del Museo Nacional de Ciencias Naturales, CSIC, 12: 1-423.

Alberdi, M.T.; Prado, J.L. \& Ortiz Jaureguizar, E. (1995). Patterns of body size changes in fossil and living Equini (Perissodactyla). Biological Journal of the Linnean Society, 54: 349-370. doi:10.1111/ j.10958312.1995.tb01042.x

Alberdi, M.T.; Prado, J.L. \& Miotti, L. (2001a). Hippidion saldiasi Roth, 1899 (Mammalia, Perissodactyla) at the Piedra Museo Site (Patagonia): their implication for the regional economy and environmental. Journal of Archaeological Science, 28: 411-419. doi:10.1006/jasc.2000.0647

Alberdi, M.T.; Zárate, M. \& Prado, J.L.(2001b). Presencia de Hippidion principale en los acantilados costeros de Mar del Plata (Argentina). Revista Española de Paleontología, 16: 1-7.

Alberdi, M.T.; Prado, J.L. \& Cartelle, C. (2002). El registro de Stegomastodon (Mammalia, Gomphotheriidae) en el Pleistoceno superior de Brasil. Revista Española de Paleontología, 17(2): 217-235.

Alberdi, M.T.; Cartelle, C. \& Prado, J.L. (2003). El registro Pleistoceno de Equus (Amerhippus) e Hippidion (Mammalia, Perissodactyla) de Brasil. Consideraciones paleoecológicas y biogeográficas. Ameghiniana, 40: 173-196.

Alberdi, M.T.; Prado, J.L. \& Salas, R. (2004). The Pleistocene Gomphotheriidae (Proboscidea) from Peru. Neues Jahrbuch für Geologie und Paläontologie, Abh., 231(3): 423-452.

Alberdi, M.T.; Prado, J.L. \& Favier-Dubois, C.M. (2006). Nuevo registro de Hippidion principale (Mammalia, Perissodactyla) del Pleistoceno de Mar del Sur, Argentina. Revista Española de Paleontología, 21: 105-113.

Alberdi, M.T.; Prado, J.L.; López, P.; Labarca, R. \& Martínez, I. (2007a). Hippidion saldiasi Roth, 1899
(Mammalia, Perissodactyla) en el Pleistoceno tardío de Calama, norte de Chile. Revista Chilena de Historia Natural, 80: 157-171.

Alberdi, M.T.; Prado, J.L.; Perea, D. \& Ubilla, M. (2007b). Stegomastodon waringi (Mammalia, Proboscidea) from the Late Pleistocene of northeastern Uruguay. Neues Jarhbuch Geologie und Paläontologie, Abh., 243(2): 179-189.

Alberdi, M.T.; Cerdeño, E. \& Prado, J.L. (2008). Stegomastodon platensis (Proboscidea, Gomphotheriidae) en el Pleistoceno de Santiago del Estero, Argentina. Ameghiniana, 45(2): 257-271.

Ameghino, F. (1888). Rápidas diagnosis de algunos mamíferos fósiles nuevos de la República Argentina. Obras Completas, Buenos Aires V: 469-480.

Ameghino, F. (1889). Contribución al conocimiento de los mamíferos fósiles de la República Argentina. Actas de la Academia Nacional de Ciencias de Córdoba, 6 : $1-1027$.

Arribas, J.G.; Millán, A.; Sibilia, E. \& Calderón, T. (1990). Factores que afectan a la determinación del error asociado a la datación absoluta por TL: Fabrica de ladrillos. Boletín de la Sociedad Escuela de Minas, 13: 141-147.

Cabrera, A. (1929). Una revisión de los Mastodontes Argentinos. Revista del Museo de La Plata, 32: 61144.

Cabrera, A.L. (1976). Regiones fitogeográficas argentinas. In: Enciclopedia argentina de agricultura y jardinería (Parodi, L.R., ed.). Editorial Acmé, Buenos Aires, 2da edición, 2: 1-85.

Campbell, K.E.Jr.; Frailey, C.D. \& Romero-Pittman, L. (2000). The late Miocene gomphothere Amahuacatherium peruvium (Proboscidea: Gomphotheriidae) from Amazonian Peru: implications for the Great American Faunal Interchange. Boletín-Instituto Geológico Minero y Metalúrgico. Serie D: Estudios Regionales, 23: 1152.

Campbell, K.E.Jr.; Heizler, M.; Frailey, C.D.; RomeroPittman, L. \& Prothero, D.R. (2001). Upper Cenozoic chronostratigraphy of the southwestern Amazon Basin. Geology, 29(7): 595-598. doi:10.1130/00917613 (2001)029<0595:UCCOTS>2.0.CO;2

Campbell, K.E.Jr.; Prothero, D.R.; Romero-Pittman, L.; Hertel, F. \& Rivera, N. (2010). Amazonian magnetostratigraphy: dating the first pulse of the Great American Faunal Interchange. Journal South American Earth Science, 26: 619-626. doi:10.1016/j.jsames.2009.11.007

Cartelle, C. (1994). Tempo passado. Mamíferos do Pleistoceno em Minas Gerais. Brasilia, Palco Editorial, 132 pp.

Castellanos, A. (1938). El subsuelo de Rosario. Anales de la Sociedad Científica Argentina, 127: 3-13.

Cione, A.L. \& Tonni, E.P. (1995). Biostratigrafía y cronología del Cenozoico superior de la Región Pampeana. In: Evolución Biológica y Climática de la Región Pampeana durante los últimos cinco millones de años. Un ensayo de correlación con el Mediterráneo occidental (Alberdi, M.T.; Leone, G. \& Tonni, E.P., (eds.). 
Monografías del Museo Nacional de Ciencias Naturales, CSIC, 12: 47-74.

Cione, A.L. \& Tonni, E.P. (1999). Biostratigraphy and chronological scale of upper-most Cenozoic in the Pampean Area, Argentina. Quaternary of South America and Antarctica Peninsula, 12, 23-51

Cione, A.L.; Tonni, E.P. \& Dondas, A. (2005). A mastodont (Mammalia, Gomphotheriidae) from the Argentinian continental shelf. Neues Jarhbuch Geologie und Paläontologie, Mh., 2005(10): 614-630.

Cione, A.L.; Tonni, E.P.; Bargo, S.; Bond, M.; Candela, A.M.; Carlini, A.A.; Deschamps, C.M.; Dozo, M.T.; Esteban, G.; Goin, F.J.; Montalvo, C.I.; Nasif, N.; Noriega, J.I.; Ortiz Jaureguizar, E.; Pascual, R.; Prado, J.L.; Reguero, M.A.; Scillato-Yané, G.J.; Soibelzon, L.; Verzi, D.H.; Vieytes, E.C.; Vizcaino, S.F. \& Vucetich, M.G. (2007). Mamíferos continentales del Mioceno tardío a la actualidad en la Argentina: cincuenta años de estudios. Ameghiniana 50œ aniversario: 257278.

D’Orbigny (1842-44). Voyage dans l'Amérique Méridionale. Paleontologie. Bertrand edit., Paris \& Strassbourg, 3(4): 1-152.

Eisenmann, V.; Alberdi, M.T.; De Giuli, G. \& Staesche, U. (1988). Collected papers after the "New York International Hipparion Conference, 1981". In: Studying fossil horses, 1: Methodology. (Woodbrune, M. \& Sondaar, P., eds.). E.J. Brill, Leiden, 1-72.

Fidalgo, F. (1983). Algunas características de los sedimentos superficiales en la cuenca del Río Salado y en la Pampa ondulada. Coloquio Internacional Hidrología de Grandes Llanuras, La Plata: 1-19.

Fidalgo, F.; Colado, U.R. \& De Francesco, F.O. (1973). Sobre ingresiones marinas cuaternarias en los partidos de Castelli, Chascomús y Magdalena (Provincia de Buenos Aires). Actas $5^{\circ}$ Congreso Geológico Argentino, Córdoba 1972, 4: 225-240.

Fidalgo F.; De Francesco F. \& Pacual R. (1975). Geología superficial de la llanura bonaerense. Relatorio: Geología de la Provincia de Buenos Aires. Sexto Congreso Geológico Argentino, 103-138.

Fleming, S.J. (1970). Thermoluminescen Dating Refinement of Quartz inclusion Method. Archaeometry, 12: 133-143. doi:10.1111/j.14754754.1970.tb00016.x

Frassinetti, D. \& Alberdi, M.T. (2000). Revisión y estudio de los restos fósiles de Mastodontes de Chile (Gomphotheriidae): Cuvieronius hyodon, Pleistoceno superior. Estudios Geológicos, 56: 197-208.

Frassinetti, D. \& Alberdi, M.T. (2005). Presencia del género Stegomastodon entre los restos fósiles de Mastodontes de Chile (Gomphotheriidae), Pleistoceno superior. Estudios Geológicos, 61: 101-107.

Fucks, E. (2005). Estratigrafía y Geomorfología en el ámbito del curso inferior del Río Luján, Provincia de Buenos Aires. Tesis Doctoral, Facultad de Ciencias Naturales y Museo, Universidad Nacional de La Plata 857, (inédita), 239 p.

Fucks, E. \& De Francesco, F.O. (2003). Ingresiones marinas al norte de la ciudad de Buenos Aires. Su Ordenamiento Estratigráfico. Actas $2^{\circ}$ Congreso
Argentino de Cuaternario y Geomorfología, San Miguel de Tucumán: 101-103.

Fucks, E. \& Deschamps, C.M. (2008). Depósitos continentales cuaternarios en el noreste de la provincia de Buenos Aires. Revista de la Asociación Geológica Argentina, 63(3): 326-343.

Gray, J.E. (1821). On the natural arrangement of vertebrate animals. London Medical Reposit Review, 15: 296-310.

Gutiérrez, M.; Alberdi, M.T.; Prado, J.L. \& Perea, D. (2005). Late Pleistocene Stegomastodon (Mammalia, Proboscidea) from Uruguay. Neues Jarhbuch Geologie und Paläontologie, Mh., 2005(11): 641-662.

Hoffstetter, R. (1950). Algunas observaciones sobre los caballos fósiles de la América del Sur. Amerhippus gen. nov. Boletín Informaciones Científicas Nacionales, 3: 426-454.

Iriondo, M. (1990). Map of the South American plainsits presents state. Quaternary of South America and Antarctic Peninsula, 6: 297-308.

Iriondo, M. (1991). El holoceno en el litoral Santa Fé. Comunicaciones del Museo Provincial de Ciencias Naturales Florentino Ameghino, Santa Fé, 3(1): 1-38.

Iriondo, M. (1994). The Quaternary of Ecuador. Quaternary International, 21: 101-112. doi:10.1016/10406182(94)90024-8

Iriondo, M. (1997). Models of depostation of loess and loessoids in the Upper Quaternary of South America. Journal of South American Earth Sciences, 10: 71-79. doi:10.1016/S0895-9811(97)00006-0

Iriondo, M. (1999). Climatic Changes in the South American Plains: Records of a Continent-scale osci1lation. Quaternary International, 57/58: 117-134. doi:10.1016/S1040-6182(98)00053-6

Iriondo, M. \& Krohling, D. (1999). Los sedimentos eólicos del noreste de la llanura pampeana (Cuaternario Superior). Actas $13^{\circ}$ Congreso Geológico Argentino y $3^{\circ}$ Congreso de Exploración de Hidrocarburos, Salta, 4: 27-48.

Isla, F.; Rutter, N.; Schnack, E. \& Zárate, M.A. (2000). La trasgresión Belgranense en Buenos Aires. Una revisión a cien años de su definición. Cuaternario y Ciencias Ambientales, 1: 3-14.

Kemp, R.; Zárate, M.; Toms, P.; King, M.; Sanabria, J. \& Arguello, G. (2006). Late Quaternary paleosols, stratigraphy and landscape evolution in the Northern Pampa, Argentina. Quaternary Research, 66: 119-132. doi:10.1016/j.yqres.2006.01.001

Krohling, D. (1999). Upper Quaternary geology of the lower Carcarana Basin, North Pampa, Argentina. Quaternary International, 57/58: 135-148. doi:10.1016/S1040-6182(98)00055-X

Latrubesse, E.M.; da Silva, S.A.F.; Cozzuol, M. \& Absy, M.L: (2007). Late Miocene continental sedimentation in southwestern Amazonia and its regional significance: biotic and geological evidence. Journal of South American Earth Science, 23: 61-80. doi:10.1016/j.jsames.2006.09.021

Latrubesse, E.M.; Cozzuol, M.; da Silva-Caminha, S.A.F.; Rigsby, C.A.; Absy, M.L. \& Jaramillo, C. 
(2010). The late Miocene paleogeography of the Amazon Basin and the evolution of the Amazon River system. Earth-Science Review, 99: 99-124. doi:10.1016/j.earscirev.2010.02.005

Lund, P.W. (1840). Nouvelles recherches sur la faune fossile du Brésil. Annales Science Naturalles, 13: 310319.

Lund, P.W. (1846). Meddlelse af det Udbytte de I 1844 undersögte Knoglehuler Have avgivet til hundskaben om Brasiliens Dyreverden för sidste Jordomvaeltning. Det köngelige Danske Videnskabernes Selskabs Naturvidenskabelige og Mathematiske Afhandlinger, 12: 57-94.

MacFadden, B.J.; Cerling, T.E.; Harris, J.M. \& Prado, J. (1999). Ancient latitudinal gradients of $\mathrm{C}_{3} / \mathrm{C}_{4}$ grasses interpreted from stable isotopes of New World Pleistocene horse. Global Ecology and Biogeography, 8: 137149. doi:10.1046/j.1365-2699.1999.00127.x

Nabel, P. (1999). Transición Pleistoceno temprano medio en la Región Pampeana. $1^{\circ}$ Congreso Argentino de Cuaternario y Geomorfología, La Pampa, Actas: 4346.

Nabel, P.; Camilión, M.C.; Machado, G.; Spiegelman, A. \& Mormeneo, L. (1995). Magneto y litoestratigrafía de los sedimentos pampeanos en los alrededores de la ciudad de Baradero, provincia de Buenos Aires. Revista de la Asociación Geológica Argentina, 48(3-4): 193206.

Nabel, P.E.; Cione, A. \& Tonni, E.P. (2000). Environmental changes in the Pampean area of Argentina the Matuyama-Brunhes (C1r-C1n) Chrons boundary. Palaeogeography, Palaeoclimatology, Palaeoecology, 162: 403-412. doi:10.1016/S0031-0182(00)00140-1

Nambi, K.S.V. \& Aitken, M.J. (1986). Annual dose conversion factors for TL and ESR dating. Archaeometry, 28: 202-205. doi:10.1111/j.14754754.1986.tb00388.x

Osborn, H.F. (1936). Proboscidea. A monograph of the discovery, evolution, migration and extinction of the mastodons and elephants. 1: Moeritherioidea, Deinotheiroidea, Mastodontoidea. American Museum of Natural History, 1: 1-802.

Owen, R. (1840). The Zoology of The Voyage of H.M.S. Beagle under the command of Captain Fitzroy, R.N., during the years 1832 to 1836. Part I.- Fossil Mammalia. Ed. y superv. C. Darwin, 108.

Owen, R. (1848). Description of teeth and portions of jaws of two extinct anthracotherioid quadrupeds ... discovered ... in the Eoecene deposits on the $\mathrm{NW}$ coast of the Isle of Wight. Quarterly Journal Geological Society, London, 4: 103-141. doi:10.1111/j.14754754.1986.tb00388.x

Owen, R. (1869). On Fossil Remains of Equines from Central and South America referable to Equus conversidens, Ow., Equus tau, Ow., and Equus arcidens, Ow. Philosophical Transactions Royal Society, London, 159: 559-573. doi:10.1098/rstl.1869.0020

Pascual, R.; Ortega Hinojosa, E.J.; Gondar, D. \& Tonni, E.P. (1965). Las edades del Cenozoico mamalífero de la Argentina, con especial atención a aquéllas del territorio bonaerense. Anales de la Comisión de Investiga- ciones Científicas de la provincia de Buenos Aires, 6: 165-193.

Paunero, R.S.; Rosales, G.; Prado, J.L. \& Alberdi, M.T. (2008). Cerro Bombero: registro de Hippidion saldiasi Roth, 1899 (Equidae, Perissodactyla) en el Holoceno temprano de Patagonia (Santa Cruz, Argentina). Estudios Geológicos, 64(1): 89-98.

Pohlig, H. (1912). Sur une vieille mandibule de "Tetracaulodon ohioticum" Blum., avec défense in situ. Bulletin de la Societé Belge de Géologie, 26: 187-193.

Prado, J.L. \& Alberdi, M.T. (1994). A Quantitative Review of the Horse «Equus» from South America. Palaeontology, 37: 459-481.

Prado, J.L. \& Alberdi, M.T. (1996). A cladistic analysis of the Horses of the tribe Equini. Palaeontology, 39: 663-680.

Prado, J.L. \& Alberdi, M.T. (2008a). Restos de Hippidion y Equus (Amerhippus) procedentes de las Barrancas de San Lorenzo, Pleistoceno Tardío (Provincia de Santa Fé, Argentina). Revista Española de Paleontología, 23(2): 225-236.

Prado, J.L. \& Alberdi, M.T. (2008b). A Cladistic Analysis among Trilophodont Gomphotheres (Mammalia, Proboscidea). With Special Attention to the South American Genera. Palaleontology, 51(4): 903-915. doi:10.1111/j.1475-4983.2008.00785.x

Prado, J.L.; Alberdi, M.T. \& Reguero, M.A. (1998). El registro más antiguo de Hippidion Owen, 1869 (Mammalia, Perissodactyla) en América del Sur. Estudios Geológicos, 54: 85-91.

Prado, J.L.; Alberdi, M.T. \& Gómez, G.N. (2002). Late Pleistocene gomphotheres (Proboscidea) from the Arroyo Tapalqué locality (Buenos Aires, Argentina) and their taxonomic and biogeographic implication. Neues Jahrbuch für Geologie und Paläontologie, Abh., 225(2): 275-296.

Prado, J.L.; Alberdi, M.T.; Sánchez, B. \& Azanza, B. (2003). Diversity of the Pleistocene Gomphotheres (Gomphotheriidae, Proboscidea) from South America. Deinsea, 9: 347-363. doi:10.1016/j.quaint.2004.04.012

Prado, J.L.; Alberdi, M.T.; Azanza, B.; Sánchez, B. \& Frassinetti, D. (2005a). The Pleistocene Gomphotheriidae (Proboscidea) from South America. Quaternary Internacional, 126-128: 21-30.

Prado, J.L.; Alberdi, M.T.; Martínez, G. \& Gutiérrez, M.A. (2005b). Equus (Amerhippus) neogeus Lund, 1840 (Equidae, Perissodactyla) at Paso Otero 5 site (Argentina): Its implications for the extinction of the South American horse. Neues Jahrbuch für Geologie und Paläontologie Mh., 2005(8): 449-468.

Prado, J.L.; Sánchez, B. \& Alberdi, M.T. (2011). Ancient feeding ecology inferred from stable isotopic evidence from fossil horses in South America over the past 3 Ma. BMC ecology, 11: 15.

Riggi, J.; Hidalgo, F.; Martínez, O. \& Porro, N. (1986). Geología de los "Sedimentos Pampeanos" en el Partido de La Plata. Revista de la Asociación Geológica Argentina, 41(3-4): 316-333.

Rincón, A.D.; Alberdi, M.T. \& Prado, J.L. (2006). Nuevo registro de Equus (Amerhippus) santaeelenae 
(Mammalia, Perissodactyla) del pozo de asfalto de Inciarte (Pleistoceno Superior), estado Zulia, Venezuela. Ameghiniana, 43: 529-538.

Rincón, A.D.; Parra, G.E.; Prevosti, F.J.; Alberdi, M.T. \& Bell, C.J. (2009). A preliminary assessment of the Mammalian Fauna from the Pliocene-Pleistocene El Breal de Orocual Locality, Monaga State, Venezuela. In: Papers on Geology, Vertebrate Paleontology and Biostratigraphy, in honor of Mike O. Woodburne (B. Albright, ed.). Bulletin of the Museum of Northern Arizona, 65: 593-620.

Ringuelet, R.A. (1961). Rasgos fundamentales de la zoogeografía de la Argentina. Phycis, 22: 151-170.

Sánchez, B.; Prado, J.L. \& Alberdi, M.T. (2006). Ancient feeding, ecology and extinction of Pleistocene horses from the Pampean Region, Argentina. Ameghiniana, 43(2): 427-436.

Schnack, E.; Isla, F.; De Francesco, F. \& Fucks, E. (2005). Estratigrafía del Cuaternario Marino Tardío en la Provincia de Buenos Aires. $16^{\circ}$ Congreso Geológico Argentino, La Plata Relatorio: 159-182.

Schewenninger, J.L. (2009). Annexe 1: OSL Dates. Research Laboratory for Archaeology \& the History of Art (RLAHA). University of Oxford. In: Géo-archéologie de la transition Pléistocène-Holocène dans le nord-est pampéen (Buenos Aires, Argentine). Révision historique, stratigraphique et taphonomique. Perspectives pour le premier peuplement. (Toledo, M.J.). Thèse doctorale, MNHN, 590 p. Paris. Inédita.

Soibelzon, E.; Gasparini, G.M.; Zurita, A.E. \& Soibelzon, L.H. (2008). Análisis faunístico de vertebrados de las "toscas del Río de La Plata" (Buenos Aires, Argentina): un yacimiento paleontológico en desaparición. Revista Museo Argentino de Ciencias Naturales, 10(2): 291-308.

Toledo, M.J. (2005). Secuencias pleistocenas «lujanenses» en su sección tipo: Primeras dataciones $\mathrm{C}^{14} \mathrm{e}$ implicancias estratigráficas, arqueológicas e históricas, Luján-Jáuregui, provincia de Buenos Aires. Revista de la Asociación Geológica Argentina, 60(2): 417-424.

Toledo, M.J. (2009). Géo-archéologie de la transition Pléistocène-Holocène dans le nord-est pampéen (Buenos Aires, Argentine). Révision historique, stratigraphique et taphonomique. Perspectives pour le premier peuplement. Thèse doctorale, MNHN, 569 p. Paris. Inédita.

Tonni E.P.; Nabel P.; Cione A.L.; Etchichury M.; Tófalo R.; Scillato Yané G.; San Cristóbal J.; Carlini A. \& Vargas D. (1999). The Ensenada and Buenos Aires formations (Pleistocene) in a quarry near La Plata, Argentina. Journal of South American Earth Sciences, 12: 273-291. doi:10.1016/S08959811(99)00021-8.

Ubilla, M. (2004). Mammalian biostratigraphy of Pleistocene fluvial deposits in northern Uruguay, South America. Proceedings of the Geologists' Association, 115: 347-357.

Ubilla, M. \& Alberdi, M.T. (1990). Hippidion sp. (Mammalia, Perissodactyla, Equidae) en sedimentos del Pleistoceno Superior del Uruguay (Edad Mamífero Lujanense). Estudios Geológicos, 46: 453-464.

Zárate, M.A. (2003). Loess of southern South America. Quaternary Science Reviews, 22: 1987-2006. doi:10.1016/S0277-3791(03)00165-3

Zárate, M.A. \& Blasi, A.C. (1993). Late PleistoceneHolocene eolian deposits of the southern Buenos Aires province, Argentina: a preliminary model. Quaternary International, 17: 15-20. doi:10.1016/10406182(93)90075-Q

Zimmerman, D.W. (1971). Thermoluminescence Dating Using Fine Grain from Pottery. Archaeometry, 13: 2952. doi:10.1111/j.14754754.1971.tb00028.x

Recibido el 1 de septiembre de 2010 Aceptado el 21 de diciembre de 2010 Publicado online el 10 de noviembre de 2011 